\title{
Modulatory effect of Persea Americana oil against diethylnitrosamine-induced hepatotoxicity in rats: a proposed mechanism
}

\author{
Omayma A. R. Abozaid', Lobna M. Anees ${ }^{2^{*}}$ and Gehan R. Abdel-Hamed ${ }^{3}$
}

\begin{abstract}
Background: The purpose of this study was to investigate the effectiveness of Persea Americana (avocado) oil against diethylnitrosamine (DEN)-induced hepatotoxicity in rats.

Methods: For the induction of hepatotoxicity, DEN was administrated orally in a dose of $20 \mathrm{mg} / \mathrm{kg}$ B.wt for 6 successive weeks, and then the animals were gavaged with Persea Americana oil in a dose of $4 \mathrm{~mL} / \mathrm{kg}$ b.wt. daily for another 6 weeks. Serum caspase-3 activity and poly (ADP-ribose) polymerase-1 (PARP-1) levels were estimated; in addition to gene expressions for NADPH oxidase, inducible nitric oxide synthase (iNOS), BCl-2, and Bax were detected.

Results: The DEN-intoxicated group exhibited a remarkable increase in NADPH oxidase and iNOS expression combined with over-activation of PARP-1 and increased antiapoptotic Bcl-2 gene expression, whereas the expression of apoptotic biomarkers significantly decreased. On the other hand, treatment with Persea Americana oil significantly suppressed the elevated levels of hepatic enzymes and improved histopathological alterations in the liver. Furthermore, these groups displayed marked downregulation in NADPH oxidase and iNOS expressions. Persea Americana oil suppressed the expression of the antiapoptotic $\mathrm{BCl}-2$, activated the intrinsic mitochondrial apoptosis pathway through upregulation of pro-apoptotic Bax, and induced an obvious increase in caspase-3 activity. Moreover, Persea Americana oil administration markedly inhibited the activity of PARP-1.
\end{abstract}

Conclusions: This study indicated the promising potential of Persea Americana oil against DEN-induced hepatic injury through its anti-oxidative activity and pro-apoptotic effect via caspase activation and PARP-1 inhibition.

Keywords: Hepatotoxicity, Persea Americana (avocado) oil, PARP-1, NADPH oxidase, iNOS, Apoptotic biomarkers

\section{Background}

Diethylnitrosamine (DEN, $N$-Nitrosodiethylamine) is a potent hepatotoxicant and hepatocarcinogen. It causes a variety of hepatocellular injuries such as necrosis, DNA damage, cirrhosis, hypertrophy, and hepatic carcinoma (Katayama et al. 2003). N-nitroso compounds are considered a disaster health risk to man, these compounds were found in cheddar cheese, fried and cured food, tobacco

\footnotetext{
*Correspondence: Lobnaanis@gmail.com

${ }^{2}$ Health Radiation Research Department, National Center for Radiation Research and Technology (NCRRT), Egyptian Atomic Energy Authority, 3 Ahmed el Zomor Street, Nasr City, Cairo, Egypt

Full list of author information is available at the end of the article
}

products, also they are generated in stomach acidic conditions from nitrite precursors and amines found in additives or food constituents, pharmaceutical drugs, and agricultural chemical residues (Mittal et al. 2006; Janani et al. 2010). It was proposed that DEN yields the promutagenic adducts, O6-ethyl deoxyguanosine, and $\mathrm{O} 4$ and O6-ethyl deoxythymidine after its metabolic activation, leading to the induction of genetically altered hepatocytes during initiation (Vasquez-Garzon et al. 2013). This procedure of bio-activation is a key step in initiating carcinogenesis by subsequent oxidative stress and cell damage (Jayakumar et al. 2012). 
Poly (ADP-ribose) polymerase-1 (PARP-1), the PARP family's most abundant isoform, is a 116-kDa nuclear enzyme involved in numerous significant biological processes, such as DNA damage repair, replication, cell proliferation control, and apoptosis. PARP-1 is recognized to be incorporated into the process of restoring DNA damage by the cellular response. After PARP-1 identifies DNA damage, it is automatically altered and recruits repair factors to the sites of DNA damage. Nevertheless, cells with significantly damaged DNA have intensified the activity of PARP-1 causing high consumption of NAD+ and depleted pools of ATP. If unchecked, this activity triggers cellular necrosis due to cellular energetic collapse. This process is blocked by prompt cleavage and PARP-1 inactivation by caspase action. However, insults that initiate necrosis cause PARP-1 over-activation that proceeds unchecked due to inadequate caspase activation (Chaitanya et al. 2010), Furthermore, PARP inhibition has recently been shown to improve mitochondrial function, and this implicates the pathogenic role of PARP-1 in metabolism, fibrosis, and liver inflammation, and designates the possible therapeutic uses of PARP-1 inhibitors for inflammatory liver diseases (Mukhopadhyay et al. 2014).

Persea Americana, commonly known as avocado, is a widely consumed fruit that has been reported to exhibit medicinal effects on many diseases, including liver, skin, arthritis, obesity, and cancer, due to its high content of phytochemicals with potential chemopreventive activity. The protein content in Avocado is higher than in any other fruit by one to twofold. It has a higher phosphorous, iron, potassium, and manganese content, whereas it contains low sodium content. It is full of nutrients such as vitamins $\mathrm{C}$, E, riboflavin, thiamin, folate, and carotene in addition to nicotinic acid (Dreher et al. 2013). It is an exceptional source of monounsaturated fat and is an important source of the necessary linoleic acid (Wong et al. 2010). It contains numerous essential polysaccharides, including pectin and hemicelluloses (soluble fiber) and lignin and cellulose (insoluble fiber) (Dreher et al. 2013). Studies in animal models and humans have shown that it aids in weight management, decreases diabetes risk, normalizes levels of blood cholesterol, and is involved in hepatic metabolism (Octavio et al. 2014). In this investigation, we evaluated the efficacy of Persea Americana (PA) oil against DEN-induced hepatotoxicity in rats. Regarding the relationship between oxidative stress, apoptosis, and PARP-1, we decided to investigate the possible modulation of designed processes by Persea Americana oil.

\section{Methods}

\section{Chemicals}

DEN was purchased from Sigma Chemicals Co. (St. Louis, MO, USA). Also used kits were the rat caspase-3 ELISA kit was purchased from Biomatik (Ontario, Canada.), the rat PARP-1 ELISA kit purchased from MyBioSource (San Diego, California, USA), the TRIzol reagent from (Life Technologies, USA), the Reverse transcriptase supplied by (Invitrogen, CA, USA), the SYBR Green PCR Master Mix obtained from (Applied Biosystems, USA), and the used primers were purchased from Invitrogen, CA, USA. All other chemical substances used were of a higher analytical category.

Persea Americana (PA) Oil: De La Cruz ${ }^{\circledR}$ Avocado Oil is non-GMO, expeller-pressed, and free of preservatives, fragrances, or artificial colors. De La Cruz ${ }^{\circledR}$ is a Division of DLC Laboratories Inc. paramount, CA, USA. The oil was purchased from a local distributer in Cairo, Egypt.

\section{Animals}

Male Wister rats (weighing 110-120 g) were obtained from the Egyptian Holding Company for Biological Products and Vaccines; Cairo, Egypt. They were kept at the animal house at the National Center for Radiation Research and Technology. The animals were permitted to adapt for one week before the experiment was started. The animals were kept under standard laboratory conditions of dark/light cycle $(12 / 12 \mathrm{~h})$, at a temperature of $25 \pm 2{ }^{\circ} \mathrm{C}$, and a humidity of $60 \pm 5 \%$. The rats were kept in cages with free access to food and drinking water ad libitum. They received a standard laboratory (pellet) diet that was nutritionally adequate. The animals' handling procedures were based on the Animal Research Reporting of In Vivo Experiments (ARRIVE) guidelines, and the research was performed in compliance with the "Guide for the Care and Use of Laboratory Animals" prepared by the National Academy of Sciences and published by the National Institutes of Health (NIH publications No, 8023, revised 1978).

\section{Experimental design}

Twenty-four rats were randomly divided into four main equal groups, six animals each, placed in individual cages and classified as follows:

\section{Group (1): normal control group}

Rats obtained regular saline, as normal control groups.

\section{Group (2): DEN group}

The animals were administered orally with DEN (dissolved in $0.9 \%$ normal saline), in a dose of $20 \mathrm{mg} / \mathrm{kg}$ 
b.w. according to the method of Darwish and El-Boghdady (2011), 5 days a week for 6 weeks.

\section{Group (3): Persea Americana (PA) oil normal treated group}

Rats were administrated with PA oil $(4 \mathrm{~mL} / \mathrm{kg}$ b.wt, orally) daily for 6 weeks according to the method of Ortiz-Avila et al. (2017).

\section{Group (4): $D E N+$ Persea Americana (PA) oil treated group}

Rats were administrated with DEN (20 mg/Kg b.wt, orally) for 6 weeks of the experiment as in group 2, after the last dose of DEN rats were treated with PA oil ( $4 \mathrm{~mL}$ / $\mathrm{kg}$ b.wt, orally) daily for another 6 weeks.

By the end of the experiment time, rats were deprived of diet overnight, anesthetized with diethyl ether, and sacrificed by cervical dislocation. The blood samples were withdrawn by intra-cardiac puncture and allowed to coagulate, then centrifuged for $15 \mathrm{~min}$ at $3000 \mathrm{rpm}$ which were then stored at $-80{ }^{\circ} \mathrm{C}$ as aliquots for further analysis. The abdomen was dissected to remove the liver, part of the liver then washed in ice-cold saline, plotted to dry for biochemical studies. The second part of the liver was dissected for histopathological examination.

\section{Determination of liver function in serum}

Serum alanine aminotransferase (ALT), aspartate aminotransferase (AST), alkaline phosphatase (ALP), total protein, total and direct bilirubin were estimated using commercially available kits (Spinreact, Santa Coloma, Spain).

\section{Determination of oxidative stress markers in liver tissue homogenate}

The dissected livers were washed with saline, dried, and homogenized in ice-cold $0.1 \mathrm{M}$ phosphate buffer $(\mathrm{pH}$ 7.4) to yield a $10 \%(\mathrm{w} / \mathrm{v})$ homogenate using Teflon homogenizer Glas-Col, Terre Haute, Ind., USA. The homogenate was centrifuged at 10,000 $\mathrm{g}$ for $15 \mathrm{~min}$. Lipid peroxidative products were measured using the thiobarbituric acid test for malondialdehyde (MDA), as described by Yoshioka et al. (1979). The content of reduced glutathione (GSH) was estimated according to the Beutler et al. (1963) method.

\section{Determination of caspase-3 in liver tissue homogenate}

Determination of liver caspase- 3 levels using a rat caspase-3 enzyme-linked immunosorbent assay (ELISA) kit purchased from Biomatik, Ontario, Canada. The test principle applied in this kit is Sandwich enzyme immunoassay. In brief, $100 \mu \mathrm{L}$ of standards or samples were added to a microtiter plate wells pre-coated with a biotin-conjugated antibody specific to Caspase 3 (CASP3), incubated for $2 \mathrm{~h}$ at $37^{\circ} \mathrm{C}$, then aspirated. Next, $100 \mu \mathrm{L}$ of
Avidin conjugated to Horseradish Peroxidase (HRP) was added to each microplate well and incubated for $1 \mathrm{~h}$ at $37^{\circ} \mathrm{C}$, then aspirated and washed 3 times. After the addition of $90 \mu \mathrm{L}$ of TMB substrate solution and incubated for $10-20 \mathrm{~min}$ at $37^{\circ} \mathrm{C}$, only those wells that contain Caspase 3 (CASP3), biotin-conjugated antibody, and enzyme-conjugated Avidin will exhibit a color change. The enzymesubstrate reaction is terminated by the addition of $50 \mu \mathrm{L}$ sulfuric acid solution and the color change is measured spectrophotometrically at a wavelength of $450 \mathrm{~nm}$. The concentration of Caspase 3 (CASP3) in the samples was then determined by comparing the O.D. of the samples to the standard curve.

Determination of poly (ADP-ribose) polymerase-1 (PARP-1) in liver tissue homogenate

Liver Poly (ADP-Ribose) Polymerase-1 (PARP-1) was estimated by an enzyme-linked immunosorbent assay (ELISA) using a rat PARP-1 ELISA kit purchased from MyBioSource (San Diego, California, USA) following the manufacturer's instructions.

\section{Detection of NADPH oxidase, iNOS, Bcl2, and Bax gene expressions by reverse transcription-polymerase chain reaction (RT-PCR) in liver tissue homogenate RNA extraction and CDNA synthesis}

To study gene expression of NADPH oxidase, inducible nitric oxide synthase (iNOS), B cell lymphoma-2 protein $(\mathrm{Bcl}-2)$, and $\mathrm{Bcl}-2$ associated $\mathrm{x}$-protein (Bax) in liver tissue, total RNA was isolated from $50 \mathrm{mg}$ liver tissue using TRIzol reagent (Life Technologies, USA) RNA content and purity were measured by a UV spectrophotometer. First-strand complementary DNA (cDNA) synthesis was performed using reverse transcriptase (Invitrogen). In brief, a template of $1 \mu \mathrm{g}$ RNA was added to a $0.5 \mathrm{mg}$ random primer, $5 \times$ RT buffer, $2.5 \mathrm{mmol} / \mathrm{L} \mathrm{dNTP}, 20 \mathrm{U}$ RNase inhibitor, and $200 \mathrm{U}$ MMLV reverse transcriptase in a total volume of $25 \mathrm{ml}$, the mixture was incubated at $37^{\circ} \mathrm{C}$ for $60 \mathrm{~min}$, then heated to $95^{\circ} \mathrm{C}$ for $5 \mathrm{~min}$ to inactivate MMLV (Rashed et al. 2011).

\section{Quantitative real-time polymerase chain reaction ( $q P C R$ )}

RT-PCRs were performed in a thermal cycler step one plus (Applied Biosystems, USA) using Sequence Detection Software (PE Biosystems, CA). Table 1 lists the primers used. The reaction mixture with a total volume of $25 \mu \mathrm{L}$ consisted of 2X SYBR Green PCR Master Mix (Applied Biosystems), $900 \mathrm{nM}$ of each primer, and $2 \mu \mathrm{L}$ of cDNA. PCR thermal cycling conditions included an initial step at $95{ }^{\circ} \mathrm{C}$ for 5 min; 40 cycles at $95{ }^{\circ} \mathrm{C}$ for $20 \mathrm{~s}, 60{ }^{\circ} \mathrm{C}$ for $30 \mathrm{~s}$, and $72{ }^{\circ} \mathrm{C}$ for $20 \mathrm{~s}$. Absolute expression of mRNA of iNOS and NADPH oxidase was estimated according to Pfaffl (2001) using the comparative 
Table 1 List of primers used in PCR

\begin{tabular}{ll}
\hline Gene & Primer sequence \\
\hline iNOS & F:5'-CGAGGAGGCTGCCCTGCAGACTGG-3' \\
R:5'-CTGGGAGGAGCTGATGGAGTAGTA-3' & F: 5'-GGAAATAGAAAGTTGACTGGCCC -3' \\
R: 5'-GTATGAGTGCCATCCAGAGCAG-3' & F: 5'GTTGCCCTCTTCTACTTTG3' \\
Bax & 5'AGCCACCCTGGTCTTG3' \\
BCl2 & R' CGGGAGAACAGGGTATGA 3' \\
& F:5'CAGGCTGGAAGGAGAAGAT 3' \\
B-actin & R:5'GATCACGAGGTCAGGAGATG3' \\
&
\end{tabular}

Ct method. Calculations were performed by calculating the values of $\Delta$ cycle threshold $(\Delta \mathrm{Ct})$ by normalizing the average $\mathrm{Ct}$ value of each treatment compared to the endogenous control $\beta$-actin.

\section{Histopathological study}

Specimens from the liver of all examined groups were washed, dehydrated in ethyl alcohol ascending grades, washed with xylene, and embedded in paraffin wax. Sections were cut out into 5-6 $\mu \mathrm{m}$ in thickness and stained with Eosin and Hematoxylin (E \& H) for examination under the light microscope (Banchroft et al. 1996) at an original magnification of _x400, Images were taken with a digital camera (Nikon, ECLIPSE, TS100, Japan).

\section{Statistical analysis}

The SPSS (version 20) was used for data analysis. Oneway analysis of variance (ANOVA) was used to analyze data, followed by a post hoc test (Bonferroni) for multiple comparisons. The data were presented as mean \pm standard deviation (SD). Considering $P$ values $<0.05$ were statistically significant.

\section{Results}

\section{Biochemical studies}

To investigate the effect of Persea Americana (PA) oil on DEN-induced liver toxicity, serum biochemical analyses were performed to determine hepatic function. The activities of ALT, AST, ALP, and levels of total protein, total bilirubin, and direct bilirubin were measured in the serum of all animal groups. As shown in Table 2, DEN intoxication resulted in severe liver injury as manifested by a significant increase in serum activities of ALT, AST, and ALP, and total protein, total bilirubin, and direct bilirubin levels compared to control. The observed changes were improved by PA oil treatment after DEN administration (group 4) compared to group 2.

Exposure to DEN resulted in a significant increase in the hepatic content of MDA, the index of high lipid peroxidation as an indicator of ROS production. Moreover, DEN exaggerated this deleterious effect by the marked attenuation in the antioxidant tissue levels. Indeed, the DEN exposed group recorded an obvious depletion in the level of GSH in comparison to the normal group. In contrary, PA oil treatment was able to correct this imbalance respectively as compared to DEN group 2 (Fig. 1a, b). Administration of PA oil alone had a non-significant effect on oxidative stress indices as compared with normal rats.

The effects of PA oil treatment of DEN-treated rats on apoptotic biomarkers in liver tissue (Bcl-2, Bax, and caspase-3) are presented in Fig. 2A-C). In DEN-intoxicated rats, there was a marked upregulation in $\mathrm{Bcl}-2$ gene expression while the activity of caspase- 3 was significantly decreased concomitantly with a downregulation in the gene expression of Bax relative to standard control rats $(p<0.001)$. Interestingly, the administration of PA oil (post DEN exposure) abrogated the DEN-induced alterations in apoptotic biomarkers in comparison with DENtreated rats.

The effects of PA oil on the iNOS and NADPH oxidase mRNA-induced folding changes in liver tissue are represented in Fig. 3. The mRNA expressions of iNOS and NADPH oxidase increased significantly in DEN-treated rats compared with normal control rats. However, treatments with PA oil after the administration of DEN significantly downregulated the mRNA expressions of iNOS

Table 2 Effect of PA oil and/or DEN on liver function test in normal rats

\begin{tabular}{lllllll}
\hline & ALT (U/L) & AST (U/L) & ALP (U/L) & Total protein (gm/dl) & Total bilirubin (mg/dl) & Direct bilirubin (mg/dl) \\
\hline Group 1 (normal control) & $12.3 \pm 1.97$ & $16.3 \pm 1.86$ & $122.5 \pm 4.09$ & $5.69 \pm 0.38$ & $0.87 \pm 0.03$ & $0.24 \pm 0.02$ \\
Group 2 (DEN) & $59.2 \pm 5.49^{\mathrm{ac}}$ & $55.7 \pm 13.4^{\mathrm{ac}}$ & $280.7 \pm 36.52^{\mathrm{ac}}$ & $3.61 \pm 0.35^{\mathrm{ac}}$ & $2.25 \pm 0.45^{\mathrm{ac}}$ & $0.79 \pm 0.05^{\mathrm{ac}}$ \\
Group 3 (PA oil) & $15.7 \pm 1.86^{\mathrm{b}}$ & $10.2 \pm 1.47^{\mathrm{b}}$ & $123.8 \pm 4.7^{\mathrm{b}}$ & $5.68 \pm 0.36^{\mathrm{b}}$ & $0.87 \pm 0.04^{\mathrm{b}}$ & $0.21 \pm 0.03^{\mathrm{b}}$ \\
Group 4 (DEN + PA oil) & $33.5 \pm 3.45^{\mathrm{abc}}$ & $24.8 \pm 2.3^{\mathrm{bc}}$ & $184.7 \pm 4.76^{\mathrm{abc}}$ & $4.3 \pm 0.31^{\mathrm{abc}}$ & $1.37 \pm 0.27^{\mathrm{abc}}$ & $0.55 \pm 0.03^{\mathrm{abc}}$ \\
\hline
\end{tabular}

The data is presented as Mean \pm S.D. $(n=6)$. In the same column, "Mean values" with dissimilar superscript letters are significantly different at $(P \leq 0.05)$

${ }^{\text {a }}$ Significant to normal control group $(P \leq 0.05)$

${ }^{\mathrm{b}}$ Significant to DEN group $(P \leq 0.05)$

${ }^{\mathrm{C}}$ Significant to $\mathrm{PA}$ oil group $(P \leq 0.05)$ 


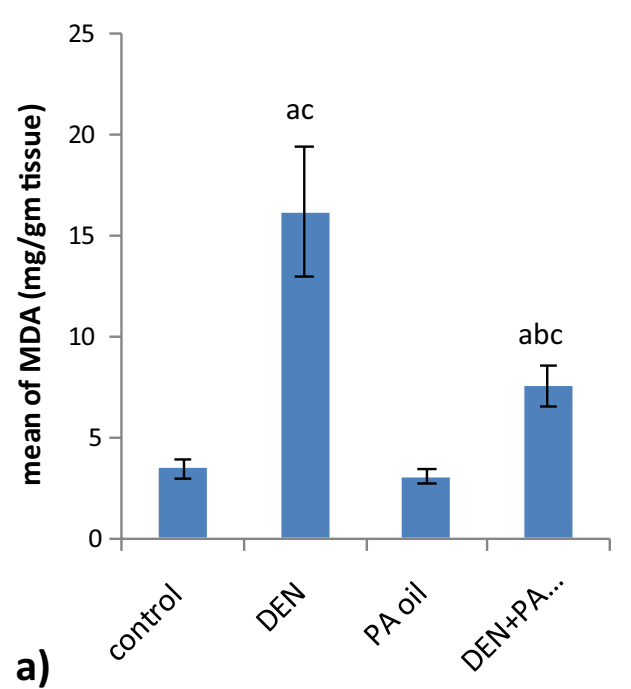

b)

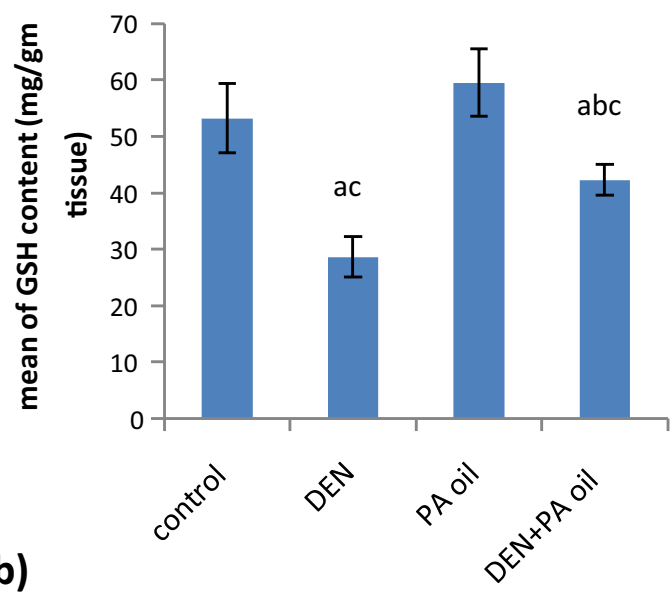

Fig. $1 \mathbf{a}, \mathbf{b}$ Effect of PA oil on liver tissue MDA and GSH content in hepatotoxicity induced in rats. Values are expressed as mean $\pm S D(n=6)$. $\mathrm{a}=$ Significant to normal control group $(P \leq 0.05), \mathrm{b}=$ Significant to DEN group $(P \leq 0.05) \mathrm{c}=$ Significant to PA oil group $(P \leq 0.05)$
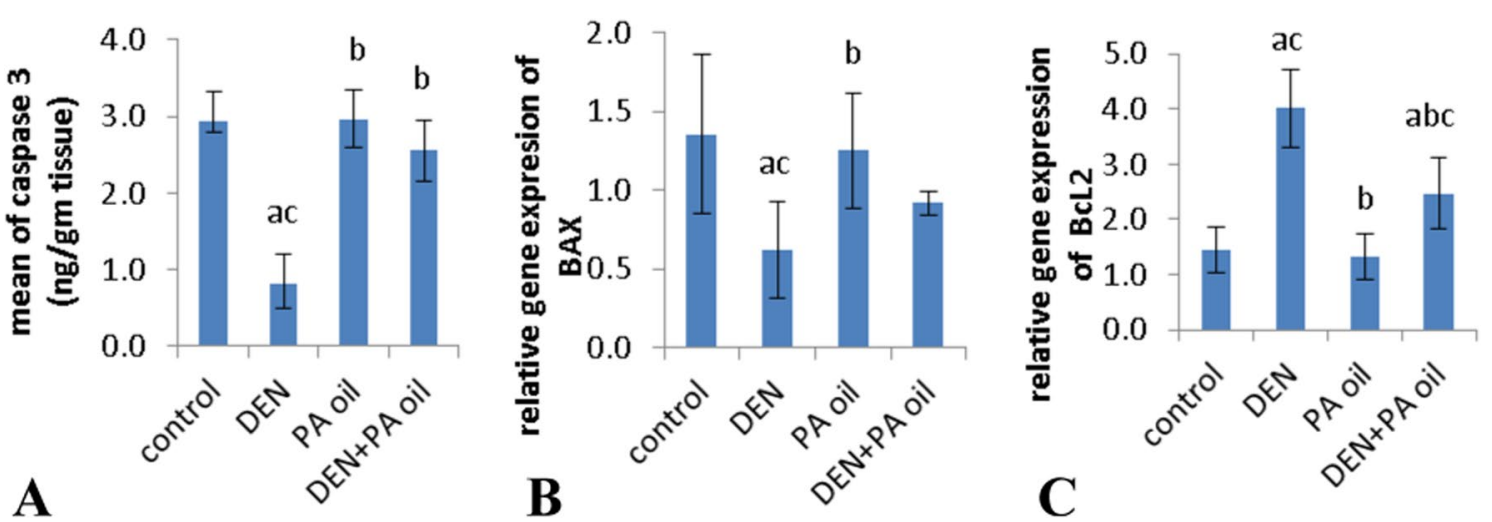

Fig.2 A-C Effect of PA oil on caspase levels in liver tissue and relative gene expression of BAX and BCL2 in rats subjected to induced hepatotoxicity. The values are expressed as mean $\pm \operatorname{SD}(n=6)$. a = Significant to normal control group $(P \leq 0.05), \mathrm{b}=$ Significant to $D E N$ group $(P \leq 0.05)$ $C=$ Significant to PA oil group $(P \leq 0.05)$

and NADPH oxidase compared with the DEN-treated rats (Fig. 3a, b).

As the generation of ROS is responsible for liver inflammatory alterations, therefore we further examined PARP-1 in the liver tissue of all the groups. A significant increase in liver PARP-1 levels was observed in DEN-treated rats when compared with the normal control group. Treatment with PA oil (post DEN exposure) exhibited a significant decrease in PARP-1 level when compared with the DEN-treated group (Fig. 4).

\section{Histopathological observation}

Histopathological examination of liver sections of control rats showed normal hepatic architecture (Fig. 5A).
Also (Fig. 5C) shows liver sections of rats received PA oil showing normal structure, while the liver portal area of rats administrated with DEN showed inflammatory cell infiltration and severe congestion in the portal vein in association with fibrosis in between the degenerated dysplastic hepatocytes all over the hepatic parenchyma (Fig. 5B). Furthermore, the treatment of rats with PA oil throughout oral gavages after DEN administration till the end of the experiment improved the hepatocellular architecture with more regular and less altered hepatocytes when compared to the DEN-treated group. The liver showed multiple dilated blood vessels with moderate leukocyte infiltration (Fig. 5D). 

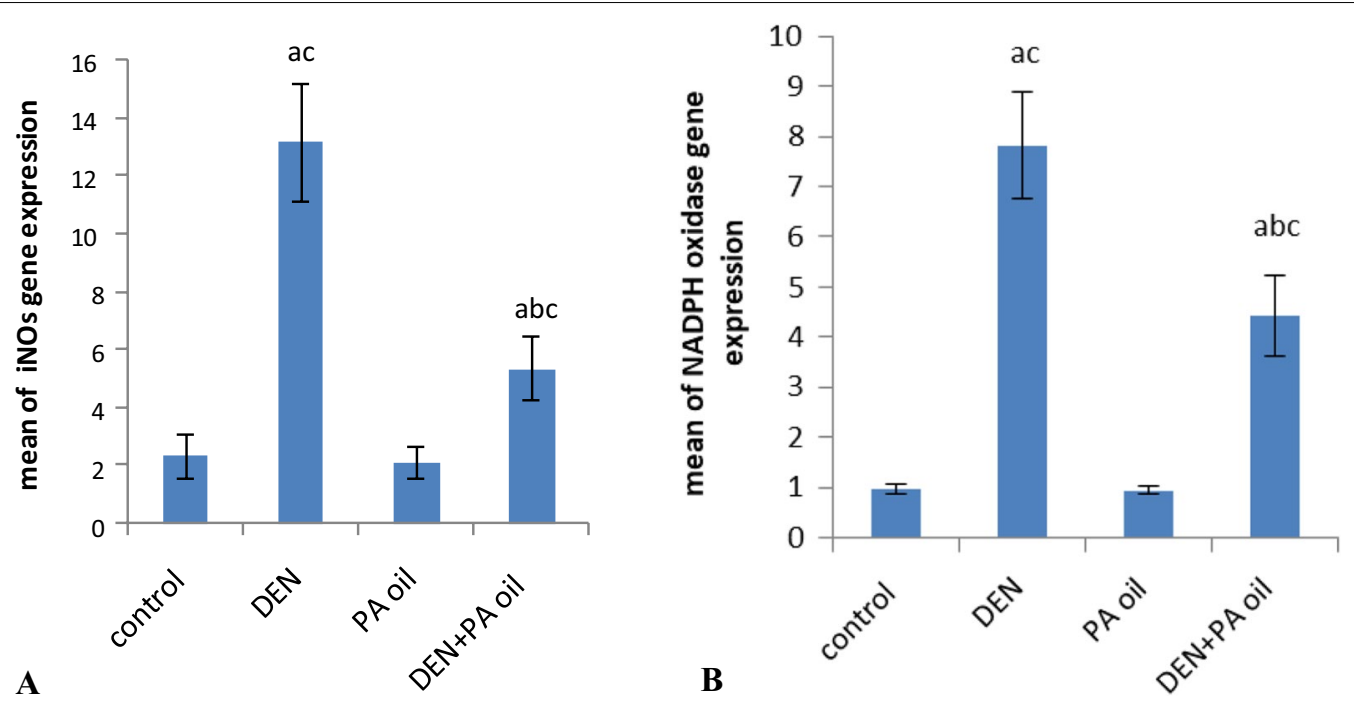

Fig. 3 a Effect of PA oil on liver tissue iNOS gene expression activities in rats subjected to induced hepatotoxicity. The values are expressed as mean \pm SD $(n=6)$. a = Significant to normal control group $(P \leq 0.05), b=$ Significant to DEN group $(P \leq 0.05) c=$ Significant to $P A$ oil group $(P \leq 0.05)$. b Effect of PA oil on liver tissue NADPH oxidase gene expression activities in rats subjected to induced hepatotoxicity. The values are expressed as mean $\pm S D(n=6)$. $a=$ Significant to normal control group $(P \leq 0.05), b=$ Significant to DEN group $(P \leq 0.05) c=$ Significant to $P A$ oil group $(P \leq 0.05)$

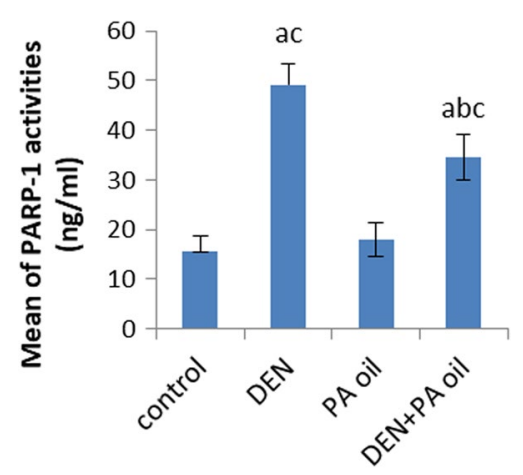

Fig. 4 Effect of PA oil on liver tissue PARP-1 concentration in rats subjected to induced hepatotoxicity. The values are expressed as mean $\pm \operatorname{SD}(n=6)$. $\mathrm{a}=$ Significant to normal control group $(P \leq 0.05)$, $\mathrm{b}=$ Significant to $D E N$ group $(P \leq 0.05) \mathrm{c}=$ Significant to $\mathrm{PA}$ oil group $(P \leq 0.05)$

\section{Discussion}

In this study, the treatment with PA oil to DEN-intoxicated rats significantly reduced the abnormalities in liver histology and caused a remarkable reduction in the elevated serum markers of liver damage, including serum ALT, AST, ALP, total and direct bilirubin, with the restoration of the reduction in serum total protein to an approximate normal level. Also, PA oil notably ameliorated the state of oxidative stress as evidenced by a significant reduction in malondialdehyde levels associated with a substantial rise in the content of reduced glutathione. Additionally, PA oil alleviated the elevation of
iNOS, NADPH oxidase gene expression, and PARP overactivation. Moreover, PA oil mitigated the reduction in caspase-3 gene activity in liver tissue. Further, PA oil elevated gene expression of suppressed pro-apoptotic Bax upon DEN intoxication and downregulated gene expression of antiapoptotic Bcl-2, thus increasing the Bax/bcl-2 ratio in favor of apoptosis rather than necrosis. Collectively, the DEN treatment could be responsible for all the phenomena of liver damage ascribed in the present work. Also, PA oil seemed to have considerable antioxidant and anti-inflammatory effects. Furthermore, it could enhance certain apoptotic pathways.

Elevations of the liver enzyme markers, including AST and ALTactivities, may indicate the existence of predominant hepatocellular disorders, whereas elevations of ALP activity may implicate predominant cholestatic diseases. In the present study, an observed enhancement in the level of hepatic serum enzymes indicates hepatocellular destruction with liver enzyme leakage after DEN administration. Similar results were obtained by (Zhao et al. 2015) who stated that DEN-intoxicated mice reported elevated levels of hepatic enzymes (ALT and AST). Hepatic secretory capacity could be evaluated by estimation of serum protein and bilirubin, the increment in bilirubin demonstrates hepatobiliary illnesses and inflammation of liver function (Pal et al. 2014), which was demonstrated in the current study. Similar findings were shown by (Carvajal-Zarrabal et al. 2014) who reported elevated levels of hepatic enzymes as well as increased serum levels of ALP and total bilirubin accompanied with 

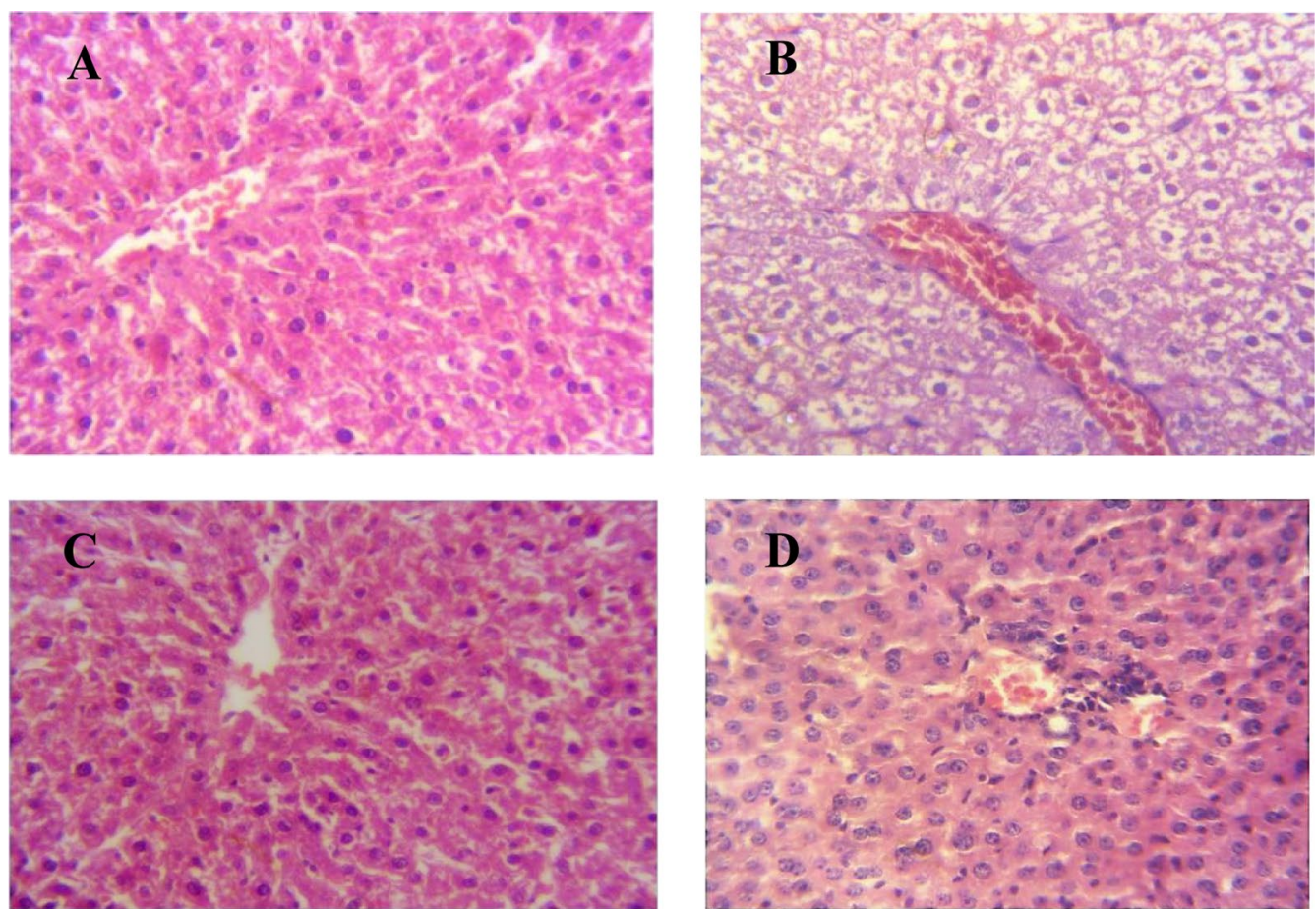

Fig. 5 A micrograph of the liver section by Light microscopy shows: A Control group; A normal hepatic architecture), normal hepatic lobules and hepatocytes B DEN-treated group; liver section of a rat intoxicated with DEN, showing congested portal vein, different degenerative changes, and necrosis of hepatic tissues around center vein (H.E. X400). C PA oil group; A liver section of a rat receiving PA oil demonstrates normal structure $(H \& E \times 400)$ D DEN + PA oil: A liver section of a rat intoxicated with DEN for 6 weeks, then treated with PA oil for another 6 weeks, shows multiple dilated blood vessels with moderate leukocyte infiltration (H\&E × 400)

decreased total protein and albumin in metabolically stressed rats.

Current results demonstrate that PA oil treatment following DEN-induced hepatotoxicity in rats exhibited a significant decrease in serum ALT, AST, and ALP when compared with the DEN group, indicating effective recovery of hepatic function may be mediated by an improvement in lipid metabolism or by delaying the onset of the hepatic disease. Consistent with the present data, Mahmoed and Rezq (2013) reported diminished hepatic enzymes ALT, AST, and ALP also reduced mortality following administration of PA oil due to inhibition of hepatocellular apoptosis in septic mice. Consequently, PA oil enhanced hepatic functions and revealed antioxidant activity. Parallel effects have been described by CarvajalZarrabal et al. (2014) who found reduced liver enzymes after avocado oil administration, which was attributed to the high percentage of unsaturated fatty acids and the low percentage of saturated fatty acids documented in avocado oil. The present findings revealed an agreement with results stated by (Oyeyemi and Oyeyemi 2015) who reported a substantial reduction in the ALT, AST, and ALP activities in the plasma and liver of the rats. Also, PA oil treatments in group 4 showed a substantial reduction in serum total and direct bilirubin relative to group 2, which could be attributed to the antioxidant capacity of the avocado oil which countered the deleterious effects of DEN on serum bilirubin (Attia and Nasr 2009).

The impaired hepatic functions may be attributed to the oxidative tissue damage caused by over-production of ROS and disruption of protective physiological molecules (as antioxidant protection mechanisms) leading to lipid peroxidation, a process that causes destruction of the macromolecules in membranes (Li et al. 2015) which play an essential role in hepatic injury pathogenesis in response to DEN administration. In this study, DEN administration resulted in a significant decrease in GSH levels with a significant increase in MDA. Mansour et al. (2019) reported that DEN metabolism induces the production of lipid peroxidation products due to the generation of abundant ROS. Further, PA oil treatment enhanced GSH levels in the intoxicated group owing to its ability to decrease ROS levels and lipid peroxidation. Ortiz-Avila et al. (2017) reported that avocado oil boosts the GSH/GSSG ratio and counteracts a persistent rise in ROS levels and intensified lipid peroxidation in diabetic rats, and protected mitochondrial membranes. Its antioxidant capacity may be also related to its constituents 
of fatty acids since monounsaturated fatty acids, such as oleic acid, which comprises $\sim 60 \%$ of the fatty acids present in the oil, are less susceptible to damage by ROS than polyunsaturated fatty acids (Wong et al. 2010). It is also conceivable that several antioxidant compounds in avocado, such as chlorophyll, tocopherols, carotenoids, and vitamins, exist in the oil and that all of these components may account for the detected effect. (Bhuyan et al. 2019).

In the present study, the administration of DEN to rats resulted in a significant increase of iNOS and NADPH oxidases gene expression compared with the control group, which is an integral player in oxidative/nitrosative stress in the systemic inflammatory response and is a typical feature of the inflammation process. This result is in agreement with Moreira et al. (2015) who reported that iNOS expression increased in a multistage model of liver carcinogenesis induced by DEN in rats. Also, in agreement with the findings of Unsal and Kurutaș (2017) the cytotoxicity and carcinogenicity of the DEN administration were attributed to its ability to induce oxidative stress and toxicity. DEN intoxication triggers activation of iNOS, which induces endogenous NO production which triggers liver damage and inflammation. The deleterious effects of $\mathrm{NO}$ may be ascribed to its nonenzymatic reaction with superoxide anion $\left(\mathrm{O}_{2}{ }^{-}\right)$, which yields the reactive nitrogen species peroxynitrite $\left(\mathrm{ONOO}^{-}\right)$. $\mathrm{ONOO}^{-}$can react with amino acids and cause protein oxidation. Additionally, $\mathrm{ONOO}^{-}$can oxidize nuclear DNA and cause nitration of tyrosine and other aromatic amino acids to generate 3-NT, a biomarker of oxidative damage and inflammation inflicted by $\mathrm{NO}$-derived $\mathrm{ONOO}^{-}$(Bishayee et al. 2010).

On the other hand, PA oil ameliorated the effect of DEN through downregulation of iNOS expression, which may substantially modulate the oxidative-induced inflammatory responses in liver tissue. Kim et al. (2000a) reported that avocado oil caused inhibition of inducible NO synthetase (iNOS), which is an inducible enzyme involved in inflammatory tissues and demonstrated to overexpress in laboratory cancer cell lines and in vivo tumors. Bishayee et al. (2010) and Kim et al. (2000b) have demonstrated the protective effect of an avocado constituent (persenon A, B) via different proposed mechanisms, such as the inhibition of nitric oxide generation is at least partially due to attenuation of iNOS protein expression and suppression of other inflammatory products, thus it may be a possible agent to prevent inflammation-associated diseases, including cancer.

Apoptosis is frequently related to a well-ordered sequence of cellular actions, which results in intracellular signaling pathway modification. To elucidate the possible proapoptotic effect of PA oil, we performed several analyses on the apoptotic pathway. PARP and caspase-3 status have been assessed as final apoptosis markers.

Regarding the apoptotic biomarkers, we estimated the execution caspase- 3 activity, expression of proteins in the Bcl-2 family, which can be classified into two categories, the pro-apoptotic Bax and Bcl-2, which is an antiapoptotic protein. These proteins are associated with mitochondrial apoptotic pathway control. The obtained data revealed that there was a significant decrease in caspase-3 activity, downregulation of Bax gene expression accompanied by significantly elevated gene expression of $\mathrm{Bcl} 2$ in DEN-induced liver damage in rats relative to the control group. This is consistent with Soha et al. (2018) and Su et al. (2019).

We found that upon treatment with PA oil, it induced caspase-3 activity, expanded gene expression of Bax, and suppressed gene expression of $\mathrm{Bcl}-2$, suggesting that these proteins were aggravated by PA oil and were involved in the apoptosis of damaged hepatocytes in DEN-intoxicated rats. Additionally, the Bax/Bcl-2 ratio was higher in PA oil supplemented groups compared to DEN-treated animals. The present findings might consistently approve the previous findings of Vivian et al. (2013) who investigated the effect of Tetrandrine on the caspase pathway. Indeed, Raina et al. (2020) reviewed different examined flavonoids demonstrating their effects against oxidative stress and apoptosis that downregulated $\mathrm{Bcl}-2$ expression and upregulated Bax expression, hence increasing the $\mathrm{Bax} / \mathrm{Bcl}-2$ ratio, thus evoking apoptosis.

In this study, we observed activation of PARP-1 expression in DEN-treated rats compared with normal control. Pathological over-activation of PARP in DEN-treated animals may be due to reactive oxygen and nitrogen species formation, mediated by iNOS and NADPH oxidases, which promote cell death and stimulate pro-inflammatory mediator production. This is consistent with the studies of Pacher and Szabó (2005), and Varga et al. (2015).

On the other hand, PARP-1 overexpression was significantly inhibited by treatment with PA oil, which due to its antioxidant capacity activated caspase to utilize PARP as its substrate and induced cleavage of PARP-1, suggesting that this is also a caspase-dependent pathway involved in the mechanism of PA oil-induced apoptosis. This result came in agreement with Moreira et al. (2015) who detected a decline in the expression of cleaved PARP in DEN-intoxicated animals which was significantly increased by treatment with melatonin. In the same context, Mukhopadhyay et al. (2014) demonstrated that PARP inhibitors facilitate the recovery of mitochondrial and various metabolic functions and facilitate the recovery of the liver after established liver fibrosis. 


\section{Conclusions}

It can be concluded that PA oil administered during inflammation or progression of hepatic disease acts as an antioxidant and inflammatory agent via downregulation of iNOS, NADPH oxidase gene expression, and PARP activity. Furthermore, it could induce upregulation of apoptotic markers to facilitate the recovery of the liver after established liver fibrosis and consequently prevent DEN-induced hepatotoxicity. However, further studies are required to determine the involvement of other signaling pathways influenced by PA oil treatment which can elucidate and potentiate the protective therapeutic applications of PA oil in hepatic injury treatment.

\section{Acknowledgements}

We wish to acknowledge the contribution of Dr. Osama Mostafa Mostafa, lecturer of Pathology, Faculty of Medicine, Aazher University, for assistance in setting up the histopathological study.

\section{Authors' contributions}

All authors OAR, LMA and GR. A-H designed the study, performed the experimental works on the rats. LMA wrote the manuscript, analyzed the data. All authors read and approved the final manuscript.

\section{Funding}

No funding

\section{Availability of data and materials}

All data generated or analyzed during this study are included in this article.

\section{Declarations}

Ethics approval and consent to participate

The current research work was performed according to the National Institutes of Health (NIH publications No, 8023, revised 1978). The animals' handling procedures were based on the Animal Research Reporting of In Vivo Experiments (ARRIVE) guidelines.

\section{Consent for publication}

Will be provided after receiving acceptance.

\section{Competing interests}

The authors declare that they have no competing interests.

\section{Author details}

${ }^{1}$ Department of Biochemistry, Faculty of Veterinary Medicine, Benha University, Benha, Egypt. ${ }^{2}$ Health Radiation Research Department, National Center for Radiation Research and Technology (NCRRT), Egyptian Atomic Energy Authority, 3 Ahmed el Zomor Street, Nasr City, Cairo, Egypt. ${ }^{3}$ Radiation Biology Department, National Center for Radiation Research and Technology (NCRRT), Egyptian Atomic Energy Authority, Cairo, Egypt.

Received: 11 September 2021 Accepted: 4 November 2021 Published online: 14 November 2021

\section{References}

Attia AM, Nasr HM (2009) Evaluation of protecrive effect of omega 3 fatty acids and selenium on paraquat intoxicated rats. Slovak J Anim Sci 42(4):180-187

Banchroft JD, Stevens A, Turner DR (1996) Theory and practice of histological techniques, 4th edn. Churchill Livingstone, New York

Beutler E, Duron O, Kelly B (1963) Improved method for the determination of blood glutathione. J Lab Clin Med 61:882-888
Bhuyan DJ, Alsherbiny MA, Perera S, Low M, Basu A, Devi OA, Barooah MS, Li CG, Papoutsis K (2019) The odyssey of bioactive compounds in avocado (Persea americana) and their health benefits. Antioxidants (Basel) 8(10):426

Bishayee A, Barnes KF, Bhatia D, Darvesh AS, Carroll RT (2010) Resveratrol suppresses oxidative stress and inflammatory response in diethylnitrosamine-initiated rat hepatocarcinogenesis. Cancer Prev Res 3(6):753-763

Carvajal-Zarrabal O, Nolasco-Hipolito C, Aguilar-Uscanga M, Melo Santiesteban G, Hayward-Jones PM, Barradas-Dermitz DM (2014) Effect of dietary intake of avocado oil and olive oil on biochemical markers of liver function in sucrose-fed rats. BioMed Res Int. https://doi.org/10.1155/2014/ 595479

Chaitanya GV, Steven AJ, Babu PP (2010) PARP-1 cleavage fragments: signatures of cell-death proteases in neurodegeneration. Cell Commun Signal $8: 31$

Darwish HA, El-Boghdady NA (2011) Possible involvement of oxidative stressin diethylnitrosamine-induced hepatocarcinogenesis: chemopreventive effect of curcumin. J Food Biochem 37(3):353-361

Dreher ML, Davenport AJ (2013) Hass avocado composition and potential health effects. Crit Rev Food Sci Nutr 53(7):738-750

Janani P, Sivakumari K, Geetha A et al (2010) Chemopreventive effect of bacoside A on $\mathrm{N}$-nitrosodiethylamine-induced hepatocarcinogenesis in rats. J Cancer Res Clin Oncol 136:759-770

Jayakumar S, Madankumar A, Asokkumar S, Raghunandhakumar S, Gokuladhas K, Kamaraj S, Divya MG, Devaki T (2012) Potential preventive effect of carvacrol against diethylnitrosamine-induced hepatocellularcarcinoma in rats. Mol Cell Biochem 360(1-2):51-60

Katayama M, Sugie S, Yoshimi N, Yamada Y, Sakata K, Qiao Z et al (2003) Preventive effect of fremented brown rice and rice bran on diethylnitrosoamine and phenobarbital induced hepatocarcinogensis in male F344 rats. Oncol Rep 10:875-80

Kim OK, Murakami A, Takahashi D (2000a) An avocado constituent, persenone A, suppresses expression of inducible forms of nitric oxide synthase and cyclooxygenase in macrophages, and hydrogen peroxide generation in mouse skin. Biosci Biotechnol Biochem 64(11):2504-2507

Kim OK, Murakami A, Nakamura Y, Takeda N, Yoshizumi H, Ohigashi H (2000b) Novel nitric oxide and superoxide generation inhibitors, persenone $A$ and B, from avocado fruit. J Agric Food Chem 48(5):1557-1563

Li S, Tan H-Y, Wang N, Zhang Z-J, Lao L, Wong C-W, Feng Y (2015) The role of oxidative stress and antioxidants in liver diseases. Int J Mol Sci 16(11):26087-26124

Mahmoed MY, Rezq AA (2013) Hepatoprotective effect of avocado fruits against carbon tetrachloride-induced liver damage in male rats. World Appl Sci J 21(10):1445-1452

Mansour DF, Abdallah HMI, Ibrahim BMM, Hegazy RR, Esmail RSE, AbdelSalam LO (2019) The carcinogenic agent diethylnitrosamine induces early oxidative stress, inflammation and proliferation in rat liver, stomach and colon: protective effect of ginger extract. Asian Pac J Cancer Prev 20(8):2551-2561

Mittal G, Brar AP, Soni G (2006) Impact of hypercholesterolemia on toxicity of N-nitrosodiethylamine: biochemical and histopathological effects. Pharmacol Rep 58:413-419

Moreira AJ, Ordoñez R, Cerski CT, Picada JN, García-Palomo A, Marroni NP et al (2015) Melatonin activates endoplasmic reticulum stress and apoptosis in rats with diethylnitrosamine-induced hepatocarcinogenesis. PLOS ONE 10(12):e0144517. https://doi.org/10.1371/journal.pone.0144517

Mukhopadhyay P, Rajesh M, Cao Z, Horváth B, Park O, Wang H et al (2014) Poly (ADP-ribose) polymerase-1 is a key mediator of liver inflammation and fibrosis. Hepatology 59(5):1998-2009

Octavio CZ, Cirilo NH, Ma AU, Guadalupe S, Patricia HJ, Dulce M (2014) Effect of dietary intake of avocado oil and olive oil on biochemical markers of liver function in sucrose-fed rats. BioMed Res Int. https://doi.org/10.1155/ 2014/595479

Ortiz-Avila O, Figueroa-García MC, García-Berumen Cl, Calderón-Cortés E, Mejía-Barajas JA, Rodriguez-Orozco AR et al (2017) Avocado oil induces long-term alleviation of oxidative damage in kidney mitochondria from type 2 diabetic rats by improving glutathione status. J Bioenerg Biomembr 49(2):205-214

Oyeyemi A, Oyeyemi R (2015) Effect of the aqueous extract of the leaves and seeds of avocado pear (Persea Americana) on some marker enzymes 
and cholesterol in the albino rat tissues. IOSR J Environ Sci Toxicol Food Technol 9:15-18

Pacher P, Szabó C (2005) Role of poly(ADP-ribose) polymerase-1 activation in the pathogenesis of diabetic complications: endothelial dysfunction, as a common underlying theme. Antioxid Redox Signal 7(11-12):1568-1580

Pal S, Bhattacharjee A, Mukherjee S, Bhattacharya K, Mukherjee S, Khowala S (2014) Effect of Alocasia indica tuber extract on reducing hepatotoxicity and liver apoptosis in alcohol intoxicated rats. Biomed Res Int. https://doi. org/10.1155/2014/349074

Pfaffl MW (2001) A new mathematical model for relative quantification in realtime RT-PCR. Nucleic Acids Res 29:2002-2007

Raina R, Hussain A, Sharma R (2020) Molecular insight into apoptosis mediated by flavones in cancer (review). World Acad Sci J. https://doi.org/10.3892/ wasj.2020.47

Rashed LA, Hashem RM, Soliman HM (2011) Oxytocin inhibits NADPH oxidase and P38 MAPK in cisplatin-induced nephrotoxicity. Biomed Pharmacother 65(7):474-480

Soha MH, Saad ME, Sahar SA (2018) CASPASE-3 and FAS-L expression and their roles as signal transduction in hepatocellular carcinoma. Bull Natl Nutr Inst Arab Repub Egypt 51(1):1-18

Su X, Zhao JQ, Li N, Kumar M, Yang AO (2019) Chemoprotective effects of resveratrol against diethylnitrosamine induced hepatocellular carcinoma in wistar rats. Int J Pharmacol 15:549-559

Unsal V, Kurutaş EB (2017) Experimental hepatic carcinogenesis: oxidative stress and natural antioxidants. Maced J Med Sci 5:1857-9655
Varga ZV, Giricz Z, Liaudet L, Haskó G, Ferdinándy P, Pacher P (2015) Interplay of oxidative, nitrosative/nitrative stress, inflammation, cell death and autophagy in diabetic cardiomyopathy. Biochim Biophys Acta 1852(2):232-242

Vasquez-Garzon VR, Macias-Perez JR, Jimenez-Garcia MN et al (2013) The chemopreventive capacity of quercetin to induce programmed cell death in hepato-carcinogenesis. Toxicol Pathol 41:857-865

Wong M, Requejo-Jackman C, Woolf A (2010) What is unrefined, extra virgin cold-pressed avocado oil. J Am Oil Chem Soc 87:1099

Yoshioka T, Kawada K, Shimada T, Mori M (1979) Lipid peroxidation in maternal and cord blood and protective mechanism against activated-oxygen toxicity in the blood. Am J Obstet Gynecol 135:372-376

Yu VWL, Ho WS (2013) Tetrandrine inhibits hepatocellular carcinoma cell growth through the caspase pathway and G2/M phase. Oncol Rep 29(6):2205-2210

Zhao X, Chen Q, Li Y, Tang H, Liu W, Yang X (2015) Doxorubicin and curcumin co-delivery by lipid nanoparticles for enhanced treatment of diethylnitrosamine-induced hepatocellular carcinoma in mice. Eur J Pharm Biopharm 93:27-36

\section{Publisher's Note}

Springer Nature remains neutral with regard to jurisdictional claims in published maps and institutional affiliations.

\section{Submit your manuscript to a SpringerOpen ${ }^{\circ}$ journal and benefit from:}

- Convenient online submission

- Rigorous peer review

- Open access: articles freely available online

- High visibility within the field

- Retaining the copyright to your article

Submit your next manuscript at $\boldsymbol{\nabla}$ springeropen.com 